\title{
Surface segregation energies in transition-metal alloys
}

\author{
Ruban, Andrei; Skriver, Hans Lomholt; Nørskov, Jens Kehlet
}

Published in:

Physical Review B Condensed Matter

Link to article, DOI:

10.1103/PhysRevB.59.15990

Publication date:

1999

Document Version

Publisher's PDF, also known as Version of record

Link back to DTU Orbit

\section{Citation (APA):}

Ruban, A., Skriver, H. L., \& Nørskov, J. K. (1999). Surface segregation energies in transition-metal alloys. Physical Review B Condensed Matter, 59(24), 15990-16000. https://doi.org/10.1103/PhysRevB.59.15990

\section{General rights}

Copyright and moral rights for the publications made accessible in the public portal are retained by the authors and/or other copyright owners and it is a condition of accessing publications that users recognise and abide by the legal requirements associated with these rights.

- Users may download and print one copy of any publication from the public portal for the purpose of private study or research.

- You may not further distribute the material or use it for any profit-making activity or commercial gain

- You may freely distribute the URL identifying the publication in the public portal 


\title{
Surface segregation energies in transition-metal alloys
}

\author{
A. V. Ruban, H. L. Skriver, and J. K. Nørskov \\ Center for Atomic-scale Materials Physics and Department of Physics, Technical University of Denmark, DK-2800 Lyngby, Denmark
}

(Received 27 August 1998)

\begin{abstract}
We present a database of $24 \times 24$ surface segregation energies of single transition metal impurities in transition-metal hosts obtained by a Green's-function linear-muffin-tin-orbitals method in conjunction with the coherent potential and atomic sphere approximations including a multipole correction to the electrostatic potential and energy. We use the database to establish the major factors which govern surface segregation in transition metal alloys. We find that the calculated trends are well described by Friedel's rectangular state density model and that the few but significant deviations from the simple trends are caused by crystal structure effects. [S0163-1829(99)05424-7]
\end{abstract}

\section{INTRODUCTION}

It is well known that the chemical composition at the surface of an alloy may differ from the composition in the bulk; that is, one of the alloy components may enrich the surface region. This phenomenon, known as surface segregation, is of vital importance in all of surface chemistry as it may enhance or suppress desirable and undesirable chemical reactions. In spite of the obvious technological interest, however, no quantitative description based on segregation energies evaluated from first-principles have been forthcoming and the present understanding of surface segregation rests to a large degree on empirical theories, even in the simplest case of a single impurity in a pure host.

The qualitative description of surface segregation is further impeded by the lack of reliable experimental data. In fact, there exists one experimental technique that yields quantitatively reliable segregation energies, namely photoemission spectroscopy of surface core-level shifts (SCLS). Within the so-called $Z+1$ approximation, a SCLS corresponds to the segregation energy of an atom of atomic number $Z+1$ in a host of atoms of atomic number $Z$. ${ }^{1}$ As a result, photoemission yields reliable surface segregation energies but only for a very restricted set of dilute alloys. For all other alloy systems one must resort to measurements of the surface composition of concentrated alloys and estimates based on the Langmuir-McLean relation between bulk and surface composition. $^{2}$

In the latter case there are two problems. First, the surface composition of most alloys is very sensitive to the external conditions and to the purity of the sample. Further, elements such as $\mathrm{H}, \mathrm{C}, \mathrm{N}, \mathrm{O}$, and $\mathrm{S}$ are, as a rule, present in all metallic systems and may easily segregate toward the surface thereby changing the surface composition due to cosegregation effects. Secondly, the Langmuir-McLean relation is only approximate and, moreover, only valid if the ordering effects in the system may be neglected. One example of a system where this condition is not fulfilled is $\mathrm{NiPt}(110)$ : According to the Langmuir-McLean relation one should observe strong Pt segregation towards the surface. Instead, the ordering effects lead to a segregation reversal. ${ }^{3}$

The dearth of experimental information leads to difficulties in the development of qualitatively reliable models. Sev- eral empirical and semiempirical theories for surface segregation in transition-metal alloys have been proposed on the basis of the Miedema theory ${ }^{4}$ and the simplest tight-binding (TB) approximation. ${ }^{5-7}$ However, these theories yield only the sign of the surface segregation energy and the comparison of these limited predictions with experiment is a rather controversial issue. In particular, the author of Ref. 8 concludes that "all examined theories fail to predict the correct segregation in a considerably high number of cases." Today, one may question this conclusion as it was reached in part on the basis of experimental data which for some systems, e.g., $\mathrm{Cu}$ and $\mathrm{Fe}$, are in conflict with the presently accepted point of view.

Unfortunately, attempts to develop a quantitative, microscopic theory based on more elaborate TB approximations $^{9-11}$ have not been particularly successful yielding results which in some cases appear even qualitatively incorrect. The first numerically derived surface segregation energies which appear quantitatively reasonable have been obtained by Foiles et al. ${ }^{12}$ who treated the first two layers of a fcc(100) surface of late transition and noble metal alloys by means of the embedded atom method. It turns out, that the application of this and similar approximate total energy methods to the earlier transition metals becomes quite complicated because of the strong nonpairwise interatomic interactions that exist in these metals and their alloys, and this kind of work has not been pursued further.

To resolve the problems mentioned above and to establish trends in the surface segregation energy for transition-metal alloys one may turn to first-principles total-energy calculations based on density-functional theory. Until recently such calculations have been quite rare and mainly connected with the determination of SCLS (Refs. 13-21) rather than segregation energies in general. In particular we note that the Green's-function linear-muffin-tin-orbitals (GF-LMTO) calculations by Alden et al. ${ }^{18,19,21}$ established systematic trends in the surface segregation energies of $Z+1$ atoms in $Z$ hosts for simple, ${ }^{19}$ rare-earth, ${ }^{21}$ and transition metals. ${ }^{18}$ These calculations revealed two important features: (i) the surface segregation energy in a transition-metal alloy is essentially given by the difference in the surface energies of the pure alloy components-a connection which has been assumed for a long time basically from common-sense arguments (see 
also Ref. 22) — and (ii) the surface segregation energies as well as the surface energies depend strongly on the crystal structure of the alloy components - a dependence noticed already by Martenson et al. ${ }^{23}$ in their analysis of experimentally determined SCLS.

The GF-LMTO calculations by Alden et al. of the segregation energies in the $Z+1$ approximation were in good agreement with the experimental observations as were the direct calculations of the surface segregation in $\mathrm{CuNi}$ and $\mathrm{NiPt}$ alloys ${ }^{24,3}$ by means of the coherent potential approximation (CPA). The success of these calculations inspired a comprehensive study by the GF-LMTO-CPA method of the segregation energies in transition-metal alloys. ${ }^{25}$ All of the above GF-LMTO calculations employed the atomic sphere approximation (ASA) with a monopole-dipole correction to the electrostatic potential and energy ${ }^{26}$ except for the work on the simple and rare earth metals where the "spherical cell model" was used. ${ }^{19,21}$ Further, in Ref. 25 all calculations were non-spin-polarized. Although the ASA including the monopole-dipole correction is quite accurate for the closepacked surfaces, i.e., fcc(111) and bcc(110), of most metals the approximation leads to unacceptably large errors in the anisotropies of the surface energies for the early transition metals.

To obtain accurate surface segregation energies for all combinations of transition metals we therefore in the present work include higher multipole moments of the electron density both in the construction of the interatomic part of the spherically symmetric one-electron potential and the electrostatic contribution to the total energy. In addition, we allow for spin-polarized solutions in all cases where magnetic hosts or impurities are involved. A detailed comparison shows that our approach leads to segregation energies that are in good agreement with full-potential GF-Korringa-Kohn-Rostoker calculations ${ }^{27}$ of single impurities in bcc Fe. A similar comparison with the measured SCLS for the $5 d$ metals shows that the present calculations leads to segregation energies which deviates by less than $0.1 \mathrm{eV}$ from the experimental values.

Using an alternative approach Drchal and co-workers ${ }^{28,29}$ have calculated the surface segregation profiles for a number of alloys including most recently the RhPt system. ${ }^{30}$ These authors rely on the general perturbation method (GPM) which completely neglects the renormalization of the host effective medium and therefore, as shown in Ref. 31, may lead to surface concentration profiles which are not only quantitatively but also qualitatively incorrect. For this reason and because Drchal and co-workers do not list surface segregation energies we will not consider their calculations here but refer to Ref. 31 where a discussion of the accuracy of the GPM is presented and to Ref. 32 where a discussion of their results for the RhPt alloy system may be found.

It is the goal of the present paper to establish the general trends in the surface segregation phenomena in transitionmetal alloys and, in particular, to discuss the reason why the simplest models do not work in all cases. To do so in a meaningful way, it is important to have a consistent set of data, experimental or theoretical, at one's disposal. It is for this reason that we devote part of the present paper, although it is not the main subject, to discuss and establish the accuracy of the database which we subsequently use to establish the trends in the surface segregation energies.

In the analysis we find that a picture based on the difference in the surface energies of the alloy components predicts a simple general behavior which is obeyed by a large proportion of the alloy systems we consider. A similar general behavior has also been found in the empirical calculations by Chelikowsky ${ }^{4}$ and Mukherjee et al. ${ }^{6}$ However, we also find large deviations from the simple behavior when the host is one of the earlier transition metals. In those cases it appears that the crystal structure of the host plays a significant role and we show how the structural contribution to the segregation energy may be accounted for in the virtual bond model.

\section{METHOD OF CALCULATION}

The surface segregation energy is the energy cost of transferring an impurity atom from the interior to the surface of a host crystal and may therefore be defined as the difference in the total energies of the system with the impurity in a surface layer and in the bulk. An alternative, but equivalent definition, is obtained from the intensive, i.e., per atom, form of the total and surface energies of the system. In this case the energy connected with the segregation of a $B$ atom from the interior of the host to the $\lambda$ th layer is given by

$$
E_{\text {segr }-\lambda}^{B}=\left.\frac{d E_{\text {surf }}\left(A_{1-c_{\lambda}} B_{c_{\lambda}}\right)}{d c_{\lambda}}\right|_{c_{\lambda}=0},
$$

where $E_{\text {surf }}\left(A_{1-c_{\lambda}} B_{c_{\lambda}}\right)$ is the surface energy of a system which consists of an $A_{1-c_{\lambda}} B_{c_{\lambda}}$ alloy embedded in the $\lambda$ th layer of an otherwise pure $A$ host and

$$
E_{\text {surf }}\left(A_{1-c_{\lambda}} B_{c_{\lambda}}\right)=\sum_{\lambda^{\prime}}\left(E_{\lambda^{\prime}}-E_{\text {bulk }}\right)-\mu c_{\lambda} .
$$

Here, $E_{\lambda^{\prime}}$ is the total energy per atom of the $\lambda^{\prime}$ th layer, $E_{b u l k}$ the total energy per atom of the host, and $\mu$ the effective chemical potential of the $B$ component in the host $A$, which is defined as

$$
\mu=\left.\frac{d E_{b u l k}\left(A_{1-c} B_{c}\right)}{d c}\right|_{c=0} .
$$

To compute the surface segregation energies from Eqs. (1)-(3) we calculated the total energies of the surface and bulk alloy using density-functional theory in the local density approximation (LDA) in conjunction with the CPA and the Green's-function technique for semi-infinite surfaces ${ }^{26,33,34}$ as implemented in the tight-binding representation $^{35-37}$ of the LMTO method. ${ }^{38,39}$ In all systems we used an spd basis set and included the core electrons in the LDA self-consistency loop. For exchange and correlation we used the Perdew-Zunger interpolation formula ${ }^{40}$ of the many-body results by Ceperley-Alder ${ }^{41}$ except when the alloy system contained one of the magnetic $3 d$ metals, $\mathrm{V}, \mathrm{Cr}$, $\mathrm{Mn}, \mathrm{Fe}, \mathrm{Co}$, and $\mathrm{Ni}$, in which case we performed spinpolarized calculations by means of the Vosko-Wilk-Nusair parametrization. $^{42}$ In the calculations for the close-packed bcc(110), hcp(0001), and fcc(111) surfaces we included eight atomic and two vacuum layers in the self-consistent iterations. For the more open surfaces these numbers were 
increased in proportion to the decreasing interlayer distance in order to keep the size of the surface region constant.

The crucial charge-transfer effects were included in the single-site approximation by the screened impurity model (SIM) (Refs. 43 and 44) giving the following correction to the electrostatic potential

$$
V_{\lambda j}^{S I M}=e^{2} q_{\lambda j} / R_{1},
$$

where $q_{\lambda j}$ is the intralayer charge transfer of the $j^{\prime}$ th alloy component, determined as the difference between its net charge, $Q_{\lambda j}$, and the average net charge of the $\lambda$ th layer, $\bar{Q}_{\Lambda}=\Sigma_{j} c_{\lambda j} Q_{\lambda j}$, i.e., $q_{\lambda j}=Q_{\lambda j}-\bar{Q}_{\lambda}$, and $R_{1}$ is the distance to the nearest coordination shell. The corresponding contribution to the electrostatic energy is

$$
E^{S I M}=-\beta \sum_{\lambda, j} c_{\lambda j} V_{\lambda j}^{S I M} q_{\lambda j},
$$

where a prefactor $\beta=0.6$ gives the best overall CPA total energies of the bulk random alloys relative to the corresponding total energies calculated either from a cluster expansion of the total energies of completely ordered alloys ${ }^{44}$ or by the super-cell approach. ${ }^{45}$ Preliminary results from super-cell calculations for surfaces of random CuPt alloys ${ }^{48}$ show that this empirical coefficient also works well at surfaces. We emphasize that this approach ensures a correct concentration dependence of the total energies and a correct renormalization of the electronic structure of the host atoms around the impurities. As a result, the chemical potentials and segregation energies may be obtained correctly in the single-site approximation. ${ }^{31}$

The second important correction to the electrostatic potential and energy in the surface calculations is the monopole contribution to the ASA potential from the higher multipole moments of the charge density and the corresponding multipole-multipole contributions to the interatomic part of the Madelung energy. The inclusion of these terms, i.e., beyond the monopole-dipole contributions, may be viewed as the first step towards the full charge-density (FCD) technique. ${ }^{46}$ Following Ref. 47 we call this correction ASA $+M$.

In the ASA $+M$ the monopole, i.e., $L=0$, contribution to the effective one-electron potential is evaluated from the multipole moments, $Q_{\lambda i}^{L}$, of the valence electron charge by the multipole expansion

$$
V_{\lambda i}^{0}=\frac{1}{S} \sum_{\nu, j, L^{\prime}} M_{\lambda i, \nu j}^{0, L^{\prime}} Q_{\nu j}^{L^{\prime}},
$$

where $S$ is the average Wigner-Seitz radius, $L$ is short hand for the $(l, m)$ quantum numbers, and $M_{\lambda i, \nu j}^{L, L^{\prime}}$ is the multipole Madelung matrix which is equivalent to the conventional (unscreened) LMTO structure constants. The corresponding Madelung contribution to the total energy is then given by

$$
E_{M}=\frac{1}{2 S} \sum_{\lambda i, L} Q_{\lambda i}^{L} \sum_{\nu j, L^{\prime}} M_{\lambda i, \nu j}^{L, L^{\prime}} Q_{\nu j}^{L^{\prime}} .
$$

A description of the procedure including expressions for the Madelung matrices and the multipole moments may be found in Ref. 26.
The number of multipoles included in the $L$ summations in Eqs. (6) and (7) is determined by the angular momentum cutoff $l_{\max }$ used in the Green's-functions calculations. Due to the properties of the Gaunt coefficients the multipole moments of the charge density have nonzero components up to $2 l_{\max }$. In the present case $l_{\max }=2$ but in the actual calculations we included Madelung contributions to the potential and the total energy only for angular momenta up to $l=3$ as the inclusion of the next momenta did not affect the results.

All calculations have been performed at the theoretically determined equilibrium volumes. For the hcp metals we used a single $c / a$ equal to 1.59 as the experimental values vary only from 1.58 to 1.61 . Lattice relaxation effects were neglected both in the bulk and surface calculations. Although the lattice relaxation contributions to the impurity solution energies and thus to the effective chemical potentials may be of the order of $0.5 \mathrm{eV}$ in unfavorable cases where the alloy components have large size difference, the effect on the surface segregation energies is very small due to the fact that the relaxation energies at the surface and in the bulk are almost equal thereby compensating each other in the final calculations. $^{25}$

\section{THE ACCURACY OF THE METHOD}

In this section we compare our calculated surface energies and surface segregation energies with the results of other calculations as well as with experimental data to establish the accuracy of the database to be presented in Sec. IV A. This is quite a difficult task since the accuracy of many of the approximations, e.g., the ASA, the single-site approximation, and the CPA, used in the present work are not well established for many of the systems we treat. Nevertheless, the comparison of our results with the relatively few calculations which do not rely on these approximations and with experimental data shows that the above-mentioned approximations lead to surface segregation energies with an accuracy of 0.1 $\mathrm{eV}$ which is sufficient to establish reliable trends. We further find that the multipole correction, i.e., ASA $+M$, is important for obtaining quantitatively correct results for surface energies as well as for surface segregation energies.

\section{A. Theoretical results}

\section{Surface energies}

In Fig. 1 we compare the surface energies for the most close-packed surfaces of the $4 d$-transition metals calculated within the ASA and the ASA $+M$ with results obtained by the FCD-LMTO method which has an accuracy similar to that found in full-potential calculations. ${ }^{49,50}$ Although the ASA, corrected only by the monopole-dipole term, ${ }^{26}$ provides a fairly good description of the trends it is seen that the surface energies of the early transition metals have errors of up to $30 \%$. It is further seen in Table I that the ASA fails to yield correct surface energy anisotropies. In contrast, the $\mathrm{ASA}+M$ yields surface energies and anisotropies which agree with the corresponding full charge-density and fullpotential results. 


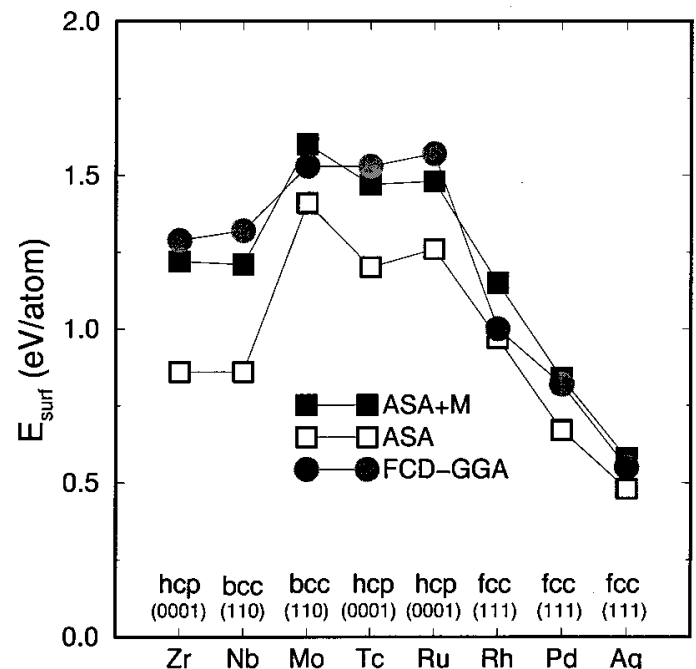

FIG. 1. The surface energies of $4 d$ transition metals calculated by the GF-LMTO technique with (ASA $+M$ ) and without (ASA) multipole correction compared with the full charge density results (FCD-GGA) (Ref. 49).

\section{Surface core-level shifts}

There exists a number of full-potential calculations of surface core-level shifts based on an ordered alloy supercell approach. ${ }^{14,15}$ For bcc(110) Mo, fcc(100) Rh, and fcc(100) Pd Methfessel et al. ${ }^{15}$ calculate the SCLS to be $-0.24 \mathrm{eV}$, $-0.65 \mathrm{eV}$, and $-0.30 \mathrm{eV}$, respectively, which compares favorably with our values of $-0.21 \mathrm{eV},-0.65 \mathrm{eV}$, and $-0.37 \mathrm{eV}$ obtained within the $\mathrm{ASA}+M$ and $Z+1$ approximations.

\section{Surface segregation energies}

Recently, Nonas et al. ${ }^{27}$ calculated the segregation energies for a series of single impurities in bcc (100) Fe by

TABLE I. Surface energies (in $\mathrm{J} / \mathrm{m}^{2}$ ) for $\mathrm{Nb}$, Mo, and Tc calculated with and without multipole correction compared to the full charge density (FCD) (Refs. 49 and 50) and full-potential (FP) results (Refs. 51 and 52).

\begin{tabular}{lcccc}
\hline \hline & ASA & ASA $+M$ & FCD & FP $^{\mathrm{a}}$ \\
\hline & \multicolumn{5}{c}{$b c c \mathrm{Nb}$} \\
$\gamma(110)$ & 1.79 & 2.53 & 2.69 & $2.362 .9^{\mathrm{b}}$ \\
$\gamma(100)$ & 1.73 & 2.88 & 2.86 & $2.863 .1^{\mathrm{b}}$ \\
$\gamma(100) / \gamma(110)$ & 0.97 & 1.14 & 1.06 & $1.211 .07^{\mathrm{b}}$ \\
& & $b c c \mathrm{Mo}$ & & \\
$\gamma(110)$ & 3.18 & 3.60 & 3.45 & 3.14 \\
$\gamma(100)$ & 2.78 & 3.97 & 3.84 & 3.52 \\
$\gamma(100) / \gamma(110)$ & 0.87 & 1.10 & 1.11 & 1.12 \\
& & $f c c \mathrm{Tc}$ & & \\
$\gamma(111)$ & 2.73 & 3.19 & 3.08 & 2.63 \\
$\gamma(100)$ & 2.87 & 3.83 & 4.05 & 3.34 \\
$\gamma(110)$ & 2.74 & 3.61 & 3.40 & 3.00 \\
$\gamma(100) / \gamma(111)$ & 1.05 & 1.20 & 1.31 & 1.27 \\
$\gamma(110) / \gamma(111)$ & 1.00 & 1.13 & 1.10 & 1.14 \\
\hline \hline
\end{tabular}

${ }^{\mathrm{a}}$ Reference 51 .

${ }^{\mathrm{b}}$ Reference 52 .
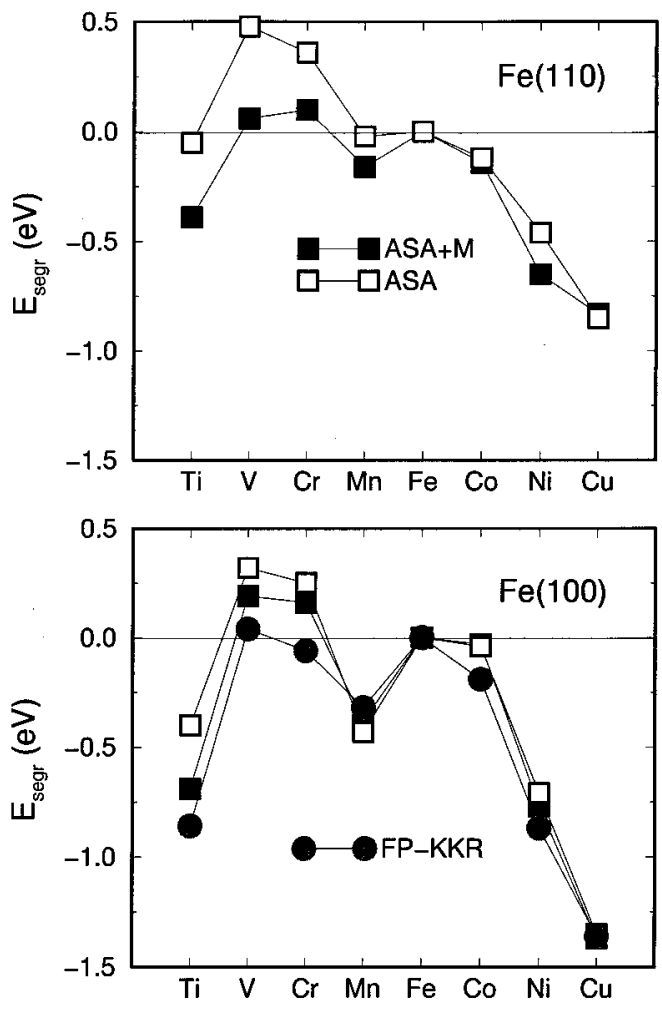

FIG. 2. The surface segregation energies of $3 d$ metals for the (110) and (100) surfaces of bcc Fe calculated with (ASA $+M)$ and without (ASA) multipole correction. The full-potential KKR results (FP-KKR) are from Ref. 27.

means of the full-potential Green's-function KKR method. The results of these calculations are presumably the most accurate to date and may serve as a measure of the accuracy of the present LMTO-CPA approach. Hence, we compare in Fig. 2 our segregation energies obtained in the ASA as well as the ASA $+M$ for $3 d$ impurities in bcc (110) and (100) $\mathrm{Fe}$ with those of Nonas et al.

One may draw several conclusions from the result shown in Fig. 2. First, we observe that the multipole correction to the ASA is important for impurities at the beginning of transition metal series. Second, the surface segregation energies depend strongly on the surface orientation: All surface segregation energies for the (110) surface are approximately a factor of 2 smaller than the corresponding values for the (100) surface. This is in accordance with the Friedel model, which will be presented in Sec. IV B and which gives a reduction of 2.19, and also with the broken bond model which was used by Nonas et al. ${ }^{27}$ to understand their first-principles results and which gives a reduction of a factor of 2 . In both cases the decrease in surface segregation energies is caused by the increase in the number of broken impurity-host bonds from 2 for (110) surfaces to 4 for (100) surfaces. As a result, it is not meaningful to compare surface segregation energies for different surface facets as is done in Ref. 27. Third and most important for our purpose, the general agreement between the ASA $+M$ and the full-potential results is good, although there are differences of order of $0.1 \mathrm{eV}$ for $\mathrm{Ti}, \mathrm{V}$, and $\mathrm{Co}$ and $0.2 \mathrm{eV}$ for $\mathrm{Cr}$. 


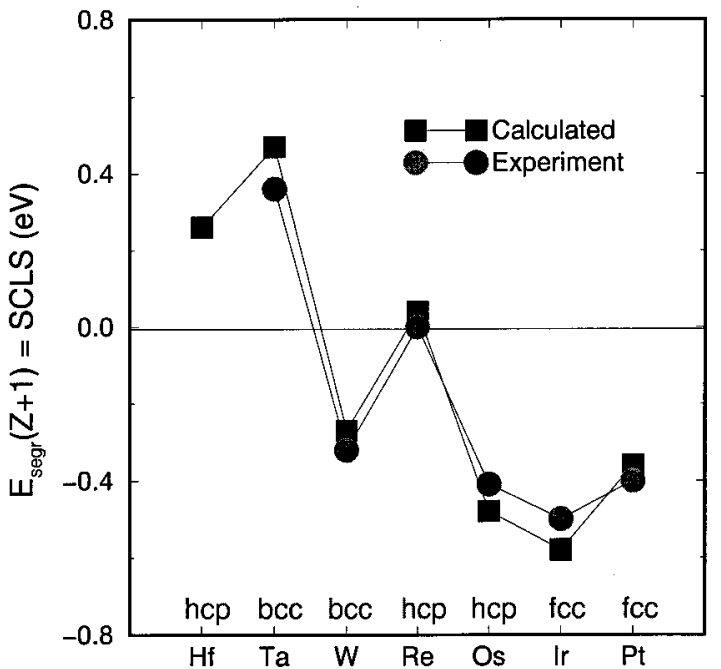

FIG. 3. The surface core-level shifts of the $5 d$ metals for the close-packed surfaces, bcc(110), fcc(111), or hcp(0001), of the experimentally observed crystal structures calculated as the surface segregation energies of $Z+1$ elements in $Z$ hosts and compared with experimental data taken from Refs. 23,55 and 56-59.

The origin of the large difference for the surface segregation energy of $\mathrm{Cr}$ in bcc (100) $\mathrm{Fe}$ is not clear at present. We have performed 96- and 216-atom supercell test calculations for $\mathrm{Fe}_{50} \mathrm{Cr}_{50}$ and for dilute alloys of $\mathrm{Cr}$ in $\mathrm{Fe}$ by the LSGF method $^{53,54}$ and they show unambiguously that both the single-site approximation and the CPA works well for this system giving accurate total energy as well as average magnetic moments of the alloy components. We may therefore only speculate that the error of $0.2 \mathrm{eV}$ for $\mathrm{Cr}$ is caused by the ASA which can lead to errors of this magnitude in the fccbcc structural energy difference for transition metals. We return to a discussion of the connection between the structural energy difference and the segregation energies in Sec. IV C. In spite of the discrepancy for $\mathrm{Cr}$ it appears that the accuracy of our ASA $+M$ approach for surface segregation energies, in general, is better than $0.1 \mathrm{eV}$ relative to the true local density result and that this accuracy is sufficient to establish a quantitatively correct picture of surface segregation in transition-metal alloys.

\section{B. Measured surface core-level shifts}

In Fig. 3 we compare our calculated surface segregation energies for $Z+1$ impurities for the close-packed surfaces of the $5 d$ metals with available experimental data for the surface core-level shifts. ${ }^{23,55-59}$ It is seen that the experimental trends are correctly reproduced by the calculation and that the agreement between theory and experiment generally is better than $0.1 \mathrm{eV}$.

In the above comparison one should note that most of the experimental values shown in the figure have been extracted from x-ray photoemission spectroscopy data neglecting the core-level shifts from the subsurface layer(s). The only exception is the data for $\mathrm{Ta}(110)$ obtained by Riffe et $a l^{56}$ who showed that there is a pronounced subsurface core-level shift of about $0.07 \mathrm{eV}$ which substantially influences the interpretation of the experimental data. The previous experimental SCLS for $\mathrm{Ta}(110),{ }^{60}$ obtained without including the subsur- face core-level shift, was therefore $0.08 \mathrm{eV}$ lower the value of $0.36 \mathrm{eV} \pm 0.012$ obtained by Riffe et al. Although our calculations still predict a somewhat higher value for the surface core-level shift, $0.47 \mathrm{eV}$, they give $0.08 \mathrm{eV}$ for the subsurface core-level shift, i.e., the segregation energy for $\mathrm{W}$ into the second layer of $\mathrm{Ta}(110)$, which is very close to the experimental value.

A similarly large subsurface segregation energy for $Z$ +1 atoms is calculated for $\mathrm{W}(110)$ and $\mathrm{Mo}(110)$ where we find $0.08 \mathrm{eV}$ and $0.12 \mathrm{eV}$, respectively. At the same time, the experimental surface core-level shifts, which are $-0.31 \mathrm{eV}$ for $\mathrm{W}(110)$ and $-0.33 \mathrm{eV}$ for $\mathrm{Mo}(110)$, have been obtained without including an additional subsurface peak in the analysis of the photoemission spectra. ${ }^{55,61}$ This may, in fact, be the reason why the experimental SCLS are lower than our theoretical values of -0.27 and $-0.21 \mathrm{eV}$, respectively. We note here that the only full-potential calculation of the SCLS in $\mathrm{Mo}(110)$ by Methfessel et al. ${ }^{14}$ give a value of $-0.24 \mathrm{eV}$ close to ours.

\section{TRENDS IN THE SURFACE SEGREGATION}

\section{A. Calculated surface segregation energies}

To help visualize the general trends we have plotted the calculated surface segregation energies in the $24 \times 24$ matrix shown in Fig. 4, color-coded according to their magnitude. In the figure, red colors correspond to negative segregation energies and, hence, to segregation of the impurity (solute) towards the surface of the host, and blue colors correspond to positive segregation energies and, hence, to the situation where the impurity prefers to remain in the interior of the host. The underlying database may be found in Table II.

A database of surface segregation energies in transitionmetal alloys has already been established by Christensen et al. ${ }^{25}$ and although the present database on account of its improved accuracy should be preferred as a source of theoretical surface segregation energies, the qualitative picture obtained from the earlier database is still correct. Further, the work of Christensen et al. ${ }^{25}$ includes a model of relaxation effects which remains valid as well as a database of the curvature of the surface energy curves which may be used to determine whether the surface alloy will form a solid solution or phase separate.

It is important to note that all the calculated segregation energies have been obtained for single impurities at closepacked surfaces and that the segregation energies at other surfaces may be quite different as demonstrated in Fig. 2. In the case of concentrated alloys the actual surface composition depends on factors such as the tendency toward ordering and the relative values of the segregation energies for different subsurface layers. In fact, even if the segregation energy of a single impurity is negative, there may be segregation reversal in a concentrated alloy, as it happens, for instance, at the (110) surface of PtNi. ${ }^{3}$ Further, due to the large difference in the reactivity of transition metals with gases such as $\mathrm{CO}, \mathrm{O}_{2}$, and $\mathrm{N}_{2}$ the surface composition of a transition-metal alloy is very sensitive to the external conditions of an experiment; see for instance Ref. 62. Hence, one should be careful when comparing the results shown in Fig. 4 with experimental data for concentrated alloys or alloys formed in deposition experiments. 


\section{Solute}
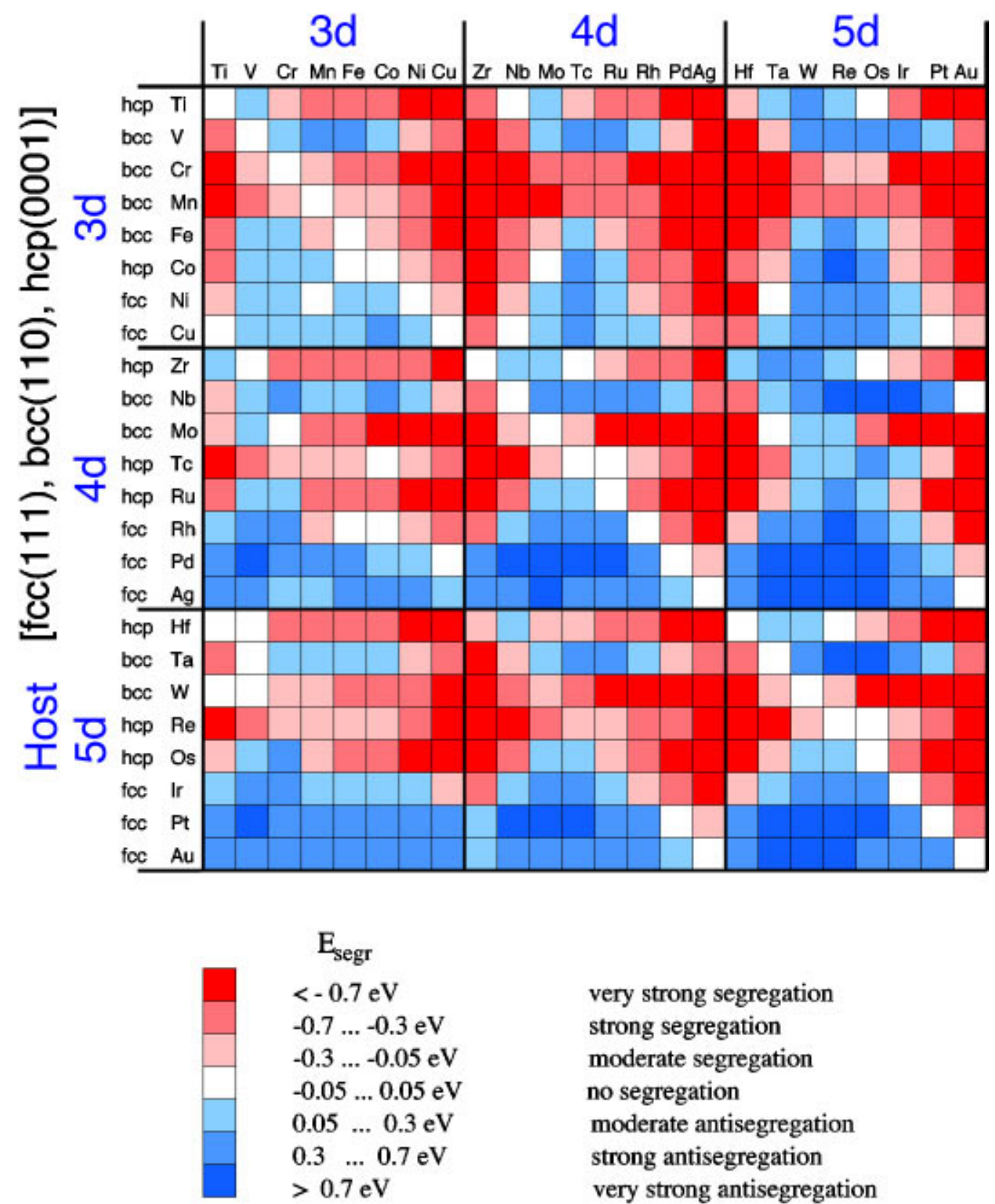

very strong segregation
strong segregation
moderate segregation
no segregation
moderate antisegregation
strong antisegregation
very strong antisegregation

FIG. 4. (Color) Surface segregation energies of transition-metal impurities (solute) for the closed-packed surfaces of transition metal hosts.

\section{B. The Friedel model for surface segregation}

It is found by Alden et al. ${ }^{18,19,21}$ that the main contribution to the surface segregation energy is given by the difference in the surface energies of the impurity and the host. This observation is confirmed by the present calculations and one may therefore attempt to use Friedel's rectangular statedensity model ${ }^{63,64}$ for surface energies to describe general trends in the surface segregation in transition-metal alloys. For this purpose we write

$$
\begin{aligned}
E_{\text {segr }}^{B \rightarrow A}= & \frac{1}{20}\left[1-\left(\frac{z_{s}}{z_{b}}\right)^{1 / 2}\right]\left\{W^{A} N_{A}\left(10-N_{A}\right)\right. \\
& \left.-W^{B \rightarrow A} N_{B}\left(10-N_{B}\right)\right\},
\end{aligned}
$$

where $z_{s}$ and $z_{b}$ are the coordination numbers at the surface and in the bulk, respectively, $N_{A}$ and $N_{B}$ are the $d$-occupation numbers of the host and the impurity, respectively, $W_{i}$ is the $d$-bandwidth of metal $i$, and $W^{B \rightarrow A}$ $=\left(W_{A} W_{B}\right)^{1 / 2}$ the $d$-bandwidth of a single $B$ impurity in the $A$ host. In Eq. (8) the square-root dependence on the coordination numbers follows from tight-binding theory and the geometric-mean dependence of $W^{B \rightarrow A}$ follows from the average bond model. ${ }^{71}$

In the simple approximation $W^{A}=W^{B \rightarrow A}$ the two terms in the curly brackets of Eq. (8) are represented by the same parabola and the model may immediately be used to construct a canonical segregation behavior. Such a construction is shown in Fig. 5 for the segregation of $4 d$ impurities in $4 d$ 


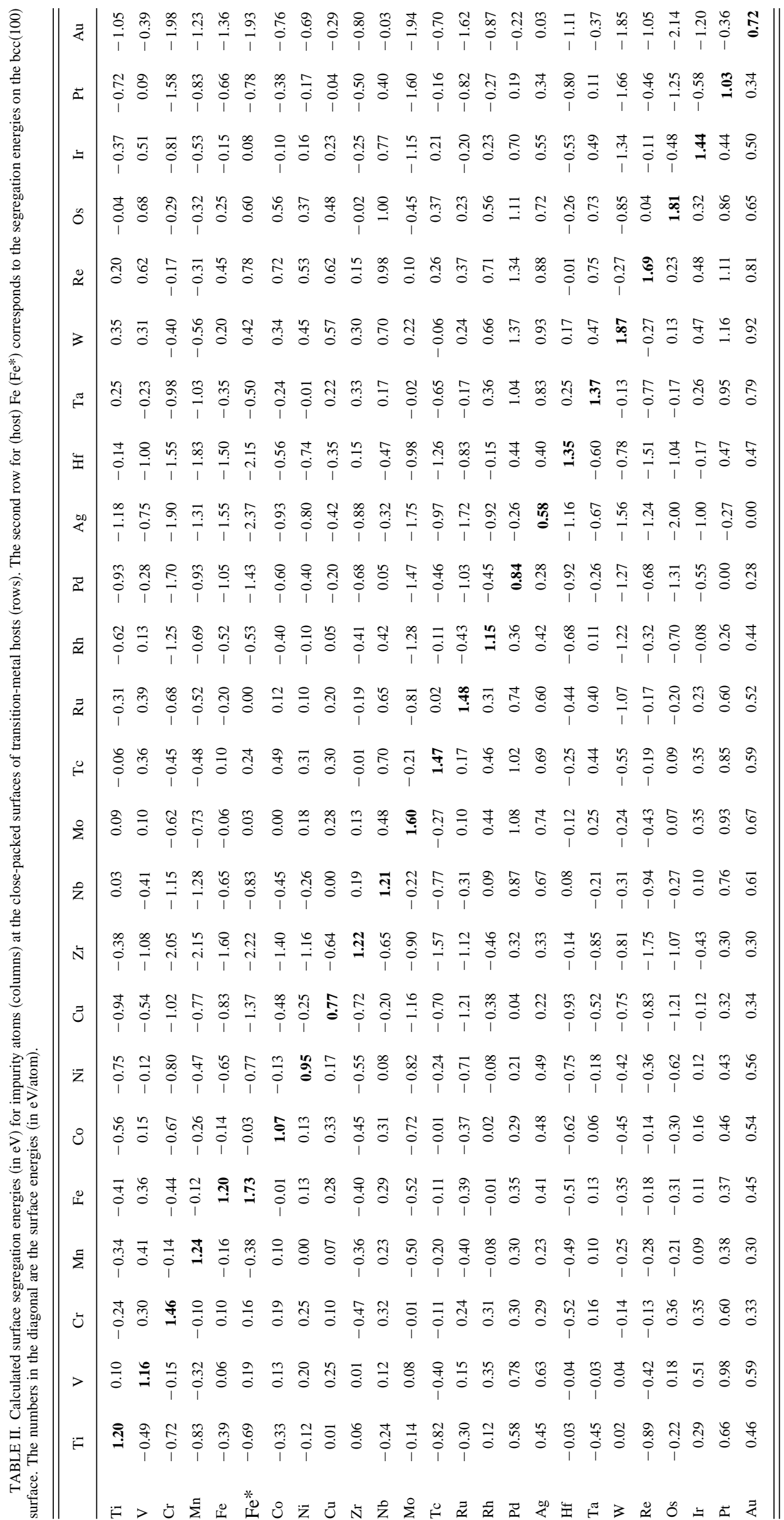



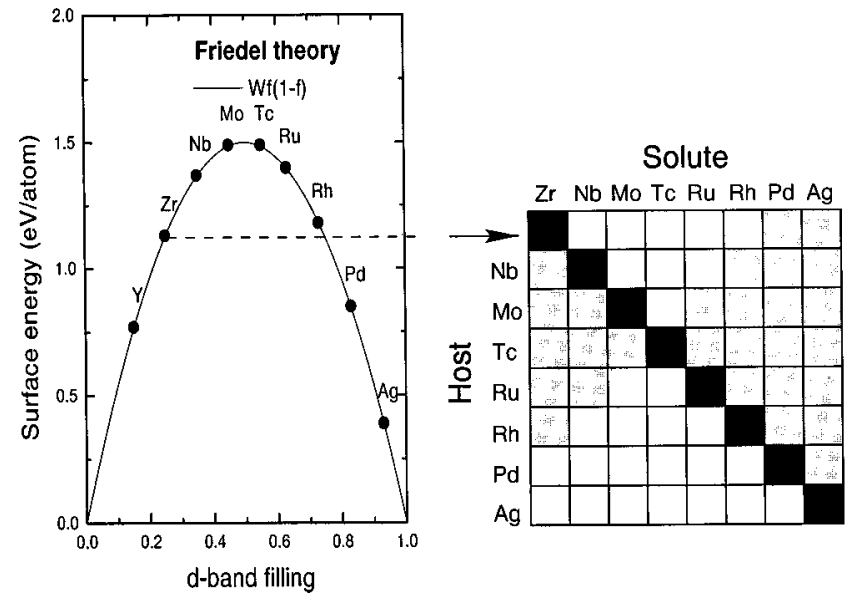

FIG. 5. Construction of the canonical segregation matrix for the $4 d \times 4 d$ metals. The parabola in the left-hand panel is the surface energy in the Friedel model given by $\gamma=w[f(f-1)]$, where $f$ is the $d$-band filling and $W=6 \mathrm{eV}$ is the $4 d$-band width. In the matrix on the right-hand side a shaded square corresponds to a negative surface segregation energy, i.e., surface segregation takes place and a white square means that surface segregation will not occur.

hosts. According to this model no impurities from the middle of a transition series is expected to segregate to the surface of early or late transition metals. Such a trend has, in fact, already been found in the empirical calculations by Chelikowsky ${ }^{4}$ and Mukherjee et al. ${ }^{6}$ which is to be expected since both of these models employ the difference in surface or cohesive energy in some form.

The "hourglass" shape of the canonical segregation matrix shown in Fig. 5 may be found to a varying degree of accuracy in each of the nine $8 \times 8$ subblocks of Fig. 4 and it appears that the Friedel model forms a meaningful starting point for the description of surface segregation in transitionmetal alloys. However, it is also clear that there are significant deviations from the canonical hourglass behavior, especially when the host is one of the first three metals in each of the series. For instance, according to Fig. 4 many metals should segregate towards the surfaces of $\mathrm{Ti}, \mathrm{Zr}$, and $\mathrm{Hf}$, but not towards the surfaces of $\mathrm{V}, \mathrm{Nb}$, and $\mathrm{Ta}$, and again towards the surfaces of the Cr, Mo, and W. This "oscillatory' behavior is clearly in contradiction to the Friedel model according to which the segregation tendency should increase monotonically from $\mathrm{Ti}, \mathrm{Zr}$, and $\mathrm{Hf}$ to $\mathrm{Cr}$, Mo, and $\mathrm{W}$ in accordance with the increasing surface energies of the impurities. In fact, the oscillatory behavior destroys the predictive power of empirical approaches. It is worth noting that a similar oscillatory behavior is exhibited by the SCLS shown in Fig. 3 and in the work of Refs. 23 and 18 where the behavior was attributed to crystal structure effects.

To demonstrate the validity and the failures of the Friedel model for surface segregation on a quantitative basis we have used Eq. (8) with $\left[1-\left(z_{s} / z_{b}\right)^{1 / 2}\right]=0.13$, which is appropriate for close-packed surfaces, and $d$ bandwidths and occupation numbers taken from Ref. 36 to calculate the surface segregation energies of $4 d$ impurities in $4 d$ transitionmetal hosts. The results are compared with the firstprinciples calculations in Fig. 6 and from this comparison it is obvious that although the Friedel model works amazingly well, in general, it cannot capture the structural dependence

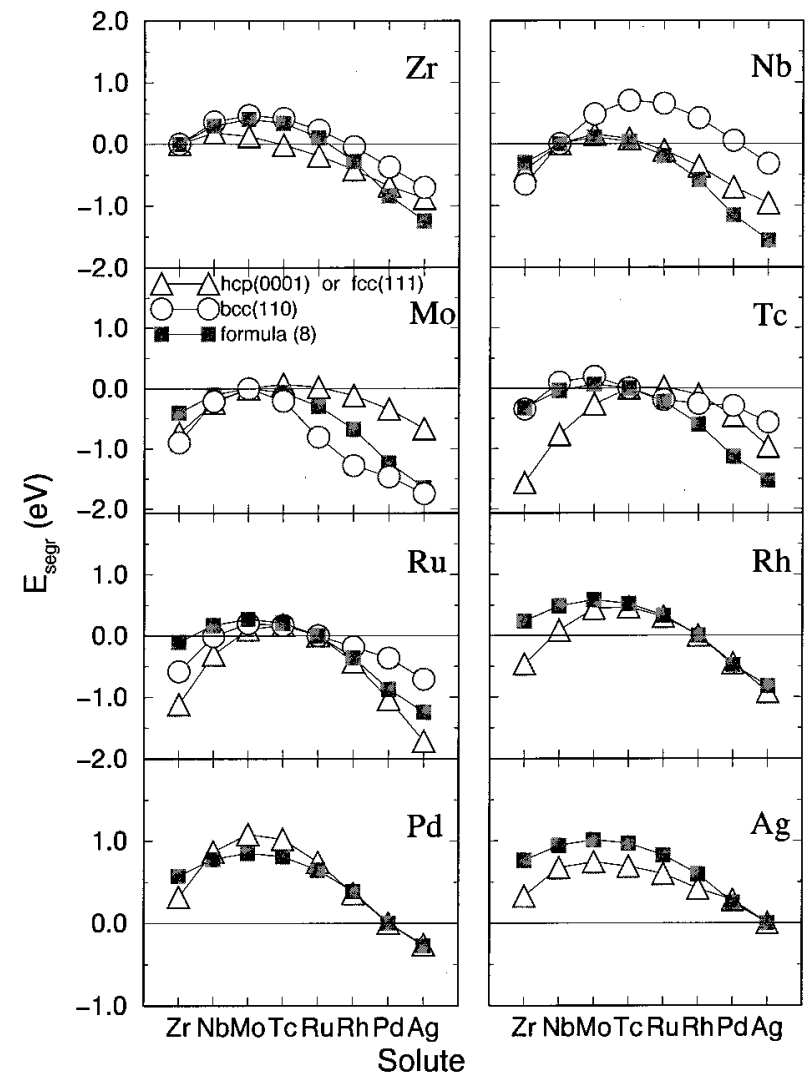

FIG. 6. The surface segregation energies for the close-packed surfaces of the $4 d$ metals. For $\mathrm{Zr}, \mathrm{Nb}$, Mo, Tc, and Ru triangles correspond to the hcp(0001) surface while for $\mathrm{Rh}, \mathrm{Pd}$, and $\mathrm{Ag}$ they correspond to $\mathrm{fcc}(111)$ surfaces. Circles correspond in all cases to bcc(110) surfaces.

of the surface segregation energy simply because the difference in the segregation energies for different crystal structures is of the same order of magnitude as the segregation energies themselves. Hence, to predict surface segregation phenomena one must take proper account of the structural contribution.

\section{Crystal structure contribution to the segregation energy}

The origin of the strong structural dependence of the surface segregation energy is the local character of the interatomic bonding in transition-metal alloys attributed to the valence $d$ electrons. The sequence of crystal structures along a transition-metal series is governed by the structural energy difference which may be considered a canonical function of the $d$ occupation number. ${ }^{65-67}$ In a tight-binding picture this canonical behavior is determined by the local atomic arrangement through the corresponding moments of the $d$ state density. ${ }^{68-70}$ The structural energy difference varies considerably along a transition series and may be as large as 1 eV/atom. If, therefore, the local atomic arrangement is disturbed either by disorder, point or other structural defects, such as impurities, vacancies, or surfaces, the system may lose or gain a substantial amount of energy depending on the crystal structure, the $d$ occupation numbers of the alloy components, and the spatial structure of the defect. 
It has recently been demonstrated that the vacancy formation energies in transition metals ${ }^{47}$ as well as the impurity solution energies in transition metal alloys ${ }^{71}$ have a substantial structural contribution. It was further shown that this structural effect could be described within a virtual bond model $^{71}$ in terms of the average $d$ occupation of the local bond. Here we show that the same local-bond model accounts for the strong structural dependence of the surface segregation energy in transition-metal alloys.

To do this, we rewrite the surface segregation energy in the form

$$
E_{\text {segr }}^{B}=\mu_{1}-\mu,
$$

where $\mu$ and $\mu_{\lambda}$ are the effective chemical potentials of $\mathrm{B}$ atoms in the bulk and in the $\lambda$ th layer at the surface of host $A$, respectively. The bulk effective chemical potential has already been defined in Eq. (3) and the definition of the surface effective chemical potential is [see Eqs. (1) and (2)]

$$
\mu_{\lambda}=\frac{d\left(\sum_{\nu} E_{\nu}\right)}{d c_{\lambda}} .
$$

As a result, the difference in the surface segregation energies of $B$ atoms in the $\alpha$ and $\beta$ structures of the host $A$ may be written

$$
\Delta E_{\text {segr }}^{\alpha-\beta}=\mu_{1}^{\alpha}-\mu_{1}^{\beta}-\mu^{\alpha}+\mu^{\beta}=\Delta \mu_{1}^{\alpha-\beta}-\Delta \mu^{\alpha-\beta} .
$$

We now determine the effective chemical potential of the bulk assuming that the dominating interactions in the system are given by interatomic pair potentials ${ }^{71}$ whereby the total energy of an $A_{1-c} B_{c}$ random alloy in the $\alpha$ structure becomes

$$
E_{A_{1-c^{B}}}^{\alpha}=v^{(0)}+(1-c)^{2} v_{A A}^{(\alpha)}+2 c(1-c) v_{A B}^{(\alpha)}+c^{2} v_{B B}^{(\alpha)} .
$$

Here, $v^{(0)}$ is the on-site term and $v_{X Y}^{(\alpha)}$ is obtained as a sum over the whole lattice of pair potentials acting between $X$ and $Y$ atoms: $v_{X Y}^{(\alpha)}=\frac{1}{2} \sum_{i \neq j} V_{X Y}\left(R_{i}-R_{j}\right)$, where $V_{X Y}$ are the structurally independent pair potentials defined, for instance, by Moriarty $^{72}$ and the sums run over the lattice sites in the $\alpha$ structure.

Using Eqs. (3) and (12) the last term in Eq. (9), i.e., the difference in the bulk effective chemical potential of $B$ atoms in a given host of different crystal structures becomes

$$
\Delta \mu^{\alpha-\beta}=2\left[\left(v_{A B}^{(\alpha)}-v_{A B}^{(\beta)}\right)-\left(v_{A A}^{(\alpha)}-v_{A A}^{(\beta)}\right)\right] .
$$

It is easy to see that the last term in Eq. (13) is the $\alpha-\beta$ structural energy difference of the host $A$, and for the first term we will use the virtual bond approximation ${ }^{71}$ assuming that interatomic potentials between $A$ and $B$ atoms $v_{A B}^{(\alpha)}$ is equal to the interatomic potential $v_{C C}^{(\alpha)}$ of a pure metal $\mathrm{C}$ with a $d$ occupation number given by $N_{d}^{C}=\frac{1}{2}\left(N_{d}^{A}+N_{d}^{B}\right)$. As a result, the difference in the chemical potentials (13) is simply the difference of the $\alpha-\beta$ structural energy difference of a hypothetical transition metal $C$ and the host $A$

$$
\Delta \mu^{\alpha-\beta}=2\left\{\Delta E_{s t r}^{\alpha-\beta}\left(\frac{1}{2}\left[N_{d}^{A}+N_{d}^{B}\right]\right)-\Delta E_{s t r}^{\alpha-\beta}\left(N_{d}^{A}\right)\right\} .
$$

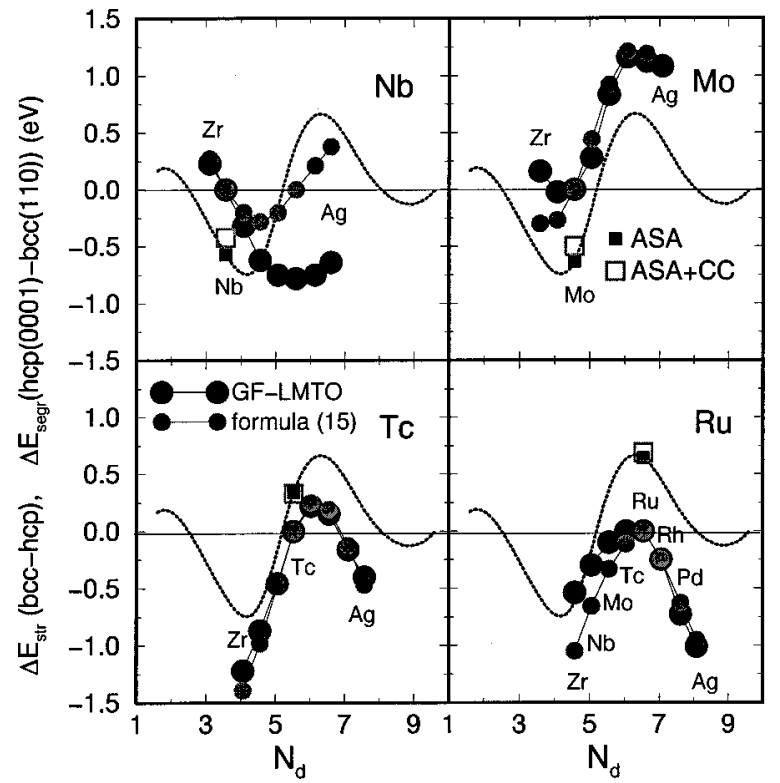

FIG. 7. The difference in the surface segregation energy for $\mathrm{hcp}(0001)$ and bcc(110) in $\mathrm{Nb}, \mathrm{Mo}$, Tc, and $\mathrm{Ru}$ calculated from first-principles (GF-LMTO) and by Eq. (8), and plotted as a function of the simple average of the impurity and host $d$ occupation number (See Ref. 71). The dotted curve is the bcc-hcp energy difference calculated for a pure elements by LMTO-ASA. For comparison we show the bcc-hcp energy difference obtained in the ASA, filled squares, and in the ASA with combined correction, open squares.

Equation (14) shows that the $\alpha-\beta$ structural difference in the chemical potentials is given solely by the $\alpha-\beta$ strucural energy difference curve. Moreover, the prefactor of 2 means that the structural energy difference, which can be of order 1 $\mathrm{eV}$, is enhanced in the crystal structure difference for the chemical potential. As has been shown in Ref. 71 this leads in some cases to a difference of about $2 \mathrm{eV}$ for the solution energies in different crystal structures of the host.

In the case of the surface segregation energy, however, there is second term $\Delta \mu_{1}^{\alpha-\beta}$ which compensates the structural difference of the bulk chemical potentials $\Delta \mu^{\alpha-\beta}$. Unfortunately, it is difficult to deduce its value from the above considerations, although (i) it is clear that for closed-packed surfaces $\Delta \mu_{\lambda}^{\alpha-\beta} \approx \Delta \mu^{\alpha-\beta}$ for $\lambda \geqslant 3$, and (ii) that, in general, $\Delta \mu_{1}^{\alpha-\beta}=\eta \Delta \mu^{\alpha-\beta}$, where $0<\eta<1$ due to the broken bonds and the destroyed crystal structure at the surface. In fact, our calculations show that $\eta=0.5$ is a perfect choice for most of the systems considered here, and thus

$$
\Delta E_{\text {segr }}^{\alpha-\beta} \approx \Delta E_{\text {str }}^{\alpha-\beta}\left(N_{d}^{A}\right)-\Delta E_{\text {str }}^{\alpha-\beta}\left(\frac{1}{2}\left[N_{d}^{A}+N_{d}^{B}\right]\right) .
$$

In Fig. 7 we compare the difference in the surface segregation energies of $4 d$ impurities on the closed-packed surfaces of bcc and hcp Nb, Mo, Tc, and Ru calculated from first principles, as well as estimated by Eq. (15) from the structural energy difference curve for the pure $4 d$ metals. In most cases the simple expression works surprisingly well. However, there are deviations, in particular, in $\mathrm{Nb}$, which we trace to the neglect in the present analysis of multiatom interactions, i.e., beyond pair potentials, which at the begin- 
ning of a transition-metal series give substantial contributions to the structural energy difference. ${ }^{73}$

In the application of Eq. (15) we made use of a structural energy difference curve calculated within the ASA. As shown in Fig. 7 this may have errors which for the bcc metals $\mathrm{Nb}$ and Mo are about $0.1 \mathrm{eV}$ compared to the more accurate calculations ${ }^{67}$ employing the combined correction term. ${ }^{38}$ Later transition metals, such as Tc and Ru, do not suffer from this kind of error. It follows from the direct connection between the surface segregation and the structural energies that the present calculations, which do not include the combined correction, for the early bcc transition metals may suffer a similar $0.1 \mathrm{eV}$ error. This may also explain the discrepances between the present and the full-potential results for spin-polarized bcc Fe in Fig. 2.

\section{SUMMARY}

We present a database of the surface segregation energies in transition-metal alloys which in contrast to a previous GFLMTO-CPA calculation include the effect of spinpolarization as well as a multipole correction to the one- electron potential and energy evaluated in the ASA. Our surface segregation energies are in good quantitative agreement with available density-functional calculations as well as experimental data. We show that a simple model based on Friedel's rectangular state-density approximation for surface energies describes the calculated trends in the surface segregation in transition metals quite well. This is in accord with the simple understanding that the alloy component which has the lowest surface energy will segregate towards the surface of the alloy. In addition, we find structural contributions as large as $1 \mathrm{eV}$ to the surface segregation energies which completely invalidates the predictions of the simple model when one of the alloy components is taken from the beginning of a transition series. We finally show that the so-called virtual bond model ${ }^{71}$ accounts for these important structural effects.

\section{ACKNOWLEDGMENTS}

The Center for Atomic-scale Materials Physics is sponsored by the Danish National Research Foundation. The present work was in part financed by The Danish Research Councils through The Center for Surface Reactivity.
${ }^{1}$ B. Johansson and N. Mårtensson, Phys. Rev. B 21, 4427 (1980).

${ }^{2} \mathrm{~J}$. M. Howe, Interfaces in Materials (John Wiley \& Sons, New York, 1997).

${ }^{3}$ I. A. Abrikosov, A. V. Ruban, H. L. Skriver, and B. Johansson, Phys. Rev. B 50, 2039 (1994).

${ }^{4}$ J. R. Chelikowsky, Surf. Sci. 139, L197 (1984).

${ }^{5}$ P. Lambin and J. P. Gaspard, J. Phys. F 10, 2413 (1980).

${ }^{6}$ S. Mukherjee and J. L. Moran-Lopez, Surf. Sci. 188, L742 (1987).

${ }^{7}$ M. Brejnak and P. Modrak, Surf. Sci. 247, 215 (1991).

${ }^{8}$ P. M. Ossi, Surf. Sci. 201, L519 (1988).

${ }^{9}$ R. Riedinger and H. Dreysse, Phys. Rev. B 27, 2073 (1983).

${ }^{10}$ H. Dreysse and R. Riedinge, Phys. Rev. B 28, 5669 (1983).

${ }^{11}$ M. C. Desjonqueres and D. Spanjaard, Phys. Rev. B 35, 952 (1987).

${ }^{12}$ S. M. Foiles, M. I. Baskes, and M. S. Daw, Phys. Rev. B 33, 7983 (1986).

${ }^{13}$ P. Feibelman, Phys. Rev. B 39, 4866 (1989).

${ }^{14}$ M. Methfessel, D. Hennig, and M. Scheffler, Surf. Sci. 287/288, 785 (1993)

${ }^{15}$ M. Methfessel, D. Hennig, and M. Scheffler, Surf. Rev. Lett. 2, 197 (1995).

${ }^{16}$ M. Alden, H. L. Skriver, and B. Johansson, Phys. Rev. Lett. 71, 2449 (1993).

${ }^{17}$ M. Alden, H. L. Skriver, and B. Johansson, Phys. Rev. Lett. 71, 2457 (1993).

${ }^{18}$ M. Alden, I. A. Abrikosov, B. Johansson, N. M. Rosengaard, and H. L. Skriver, Phys. Rev. B 50, 5131 (1994).

${ }^{19}$ M. Alden, H. L. Skriver, and B. Johansson, Phys. Rev. B 50, 12118 (1994).

${ }^{20}$ C. Engdahl, P. Stolze, K. W. Jacobsen, J. K. Nørskov, and H. L. Skriver, J. Vac. Sci. Technol. A 12, 1787 (1994).

${ }^{21}$ M. Alden, B. Johansson, and H. L. Skriver, Phys. Rev. B 51, 5386 (1995).

${ }^{22}$ G. Treglia, B. Legrand, and F. Ducastelle, Europhys. Lett. 7, 575 (1988).
${ }^{23}$ N. Mårtensson, H. B. Saafeld, H. Kuhlenbeck, and M. Neumann, Phys. Rev. B 39, 8181 (1989).

${ }^{24}$ A. V. Ruban, I. A. Abrikosov, D. Ya. Kats, D. Gorelikov, K. W. Jacobsen, and H. L. Skriver, Phys. Rev. B 49, 11383 (1994).

${ }^{25}$ A. Christensen, A. V. Ruban, P. Stolze, K. W. Jacobsen, H. L. Skriver, and J. K. Nørskov, Phys. Rev. B 56, 5822 (1997).

${ }^{26}$ H. L. Skriver and N. M. Rosengaard, Phys. Rev. B 43, 9538 (1991).

${ }^{27}$ B. Nonas, K. Wildberger, R. Zeller, and P. H. Dederichs, Phys. Rev. Lett. 80, 4574 (1998).

${ }^{28}$ A. Pasturel, V. Drchal, J. Kudrnovsky, and P. Weinberger, Phys. Rev. B 48, 2704 (1993).

${ }^{29}$ V. Drchal, J. Kudrnovsky, A. Pasturel, I. Turek, and P. Weinberger, Phys. Rev. B 54, 8202 (1996).

${ }^{30}$ V. Drchal, J. Kudrnovsky, A. Pasturel, I. Turek, P. Weinberger, A. Gonis, and P. E. A. Turchi, in Tight-Binding Approach to Computatinal Materials Science, edited by P. E. A. Turchi, A. Gonis, and L. Colombo, Mater. Res. Soc. Symp. Proc. 491 (Materials Research Society, Pittsburgh, 1998), p. 45.

${ }^{31}$ A. V. Ruban and H. L. Skriver, Phys. Rev. B 55, 8801 (1997).

${ }^{32}$ A. V. Ruban and H. L. Skriver, Comput. Mater. Sci. (to be published).

${ }^{33}$ O. Gunnarsson, O. Jepsen, and O. K. Andersen, Phys. Rev. B 27, 7144 (1983).

${ }^{34}$ I. A. Abrikosov and H. L. Skriver, Phys. Rev. B 47, 16532 (1993).

${ }^{35}$ O. K. Andersen and O. Jepsen, Phys. Rev. Lett. 53, 2571 (1984).

${ }^{36}$ O. K. Andersen, O. Jepsen, and D. Glötzel, in Highlights of Condensed-Matter Theory, edited by F. Bassani, F. Fumi, and M. P. Tosi (North-Holland, New York, 1985).

${ }^{37}$ O. K. Andersen, Z. Pawlowska, and O. Jepsen, Phys. Rev. B 34, 5253 (1986).

${ }^{38}$ O. K. Andersen, Phys. Rev. B 12, 3060 (1975).

${ }^{39}$ H. L. Skriver, The LMTO Method (Springer-Verlag, Berlin, 1984). 
${ }^{40}$ J. Perdew and A. Zunger, Phys. Rev. B 23, 5048 (1981).

${ }^{41}$ D. M. Ceperley and B. J. Alder, Phys. Rev. Lett. 45, 566 (1980).

${ }^{42}$ S. H. Vosko, L. Wilk, and M. Nusair, Can. J. Phys. 58, 1200 (1980).

${ }^{43}$ I. A. Abrikosov, Yu. H. Vekilov, P. A. Korzhavyi, A. V. Ruban, and L. E. Shilkrot, Solid State Commun. 83, 867 (1992).

${ }^{44}$ A. V. Ruban, I. A. Abrikosov, and H. L. Skriver, Phys. Rev. B 51, 12958 (1995).

${ }^{45}$ I. A. Abrikosov and B. Johansson, Phys. Rev. B 57, 14164 (1998).

${ }^{46}$ L. Vitos, J. Kollàr, and H. L. Skriver, Phys. Rev. B 55, 13521 (1997).

${ }^{47}$ P. A. Korzhavyi, I. A. Abrikosov, B. Johansson, A. V. Ruban, and H. L. Skriver, Phys. Rev. B 57, 11693 (1999).

${ }^{48}$ A. V. Ruban and H. L. Skriver (unpublished).

${ }^{49}$ L. Vitos, J. Kollár, A. V. Ruban, and H. L. Skriver, Surf. Sci. 411, 186 (1998).

${ }^{50}$ L. Vitos (private communication).

${ }^{51}$ M. Methfessel, D. Henning, and M. Scheffler, Phys. Rev. B 46, 4816 (1992).

${ }^{52}$ M. Weinert, R. E. Watson, J. W. Davenport, and G. W. Fernando, Phys. Rev. B 39, 12585 (1989).

${ }^{53}$ I. A. Abrikosov, A. M. N. Niklasson, S. I. Simak, B. Johansson, A. V. Ruban, and H. L. Skriver, Phys. Rev. Lett. 76, 4203 (1996).

${ }^{54}$ I. A. Abrikosov, S. I. Simak, B. Johansson, A. V. Ruban, and H. L. Skriver, Phys. Rev. B 56, 9319 (1996).

${ }^{55}$ D. M. Riffe, G. K. Wertheim, and P. H. Citrin, Phys. Rev. Lett. 63, 1976 (1989).

${ }^{56}$ D. M. Riffe and G. K. Wertheim, Phys. Rev. B 47, 6672 (1993).
${ }^{57}$ R. C. Baetzold, G. Apai, and E. Shustorovich, Phys. Rev. B 26, 4022 (1982).

${ }^{58}$ H. Tillborg, Ph. D. thesis, Uppsala University, 1993.

${ }^{59}$ A. Flodstrom, R. Nyholm, and B. Johansson, in Advances in Surface and Interface Science, Volume 1: Techniques, edited by R. Z. Bachrach (Plenum Press, New York, 1992).

${ }^{60}$ C. Guillot, D. Chauveau, P. Roubin, J. Lecante, and M. C. Desjonqueres, Surf. Sci. 162, 46 (1985).

${ }^{61}$ E. Lundgren, U. Johansson, R. Nyholm, and J. N. Andersen, Phys. Rev. B 48, 5525 (1993).

${ }^{62}$ D. D. Beck, C. L. DiMaggio, and G. B. Fisher, Surf. Sci. 297, 293 (1993).

${ }^{63}$ J. Friedel, Ann. Phys. (N.Y.) 1, 257 (1976).

${ }^{64}$ F. Cyrot-Lackmann, Adv. Phys. 16, 393 (1967).

${ }^{65}$ D. G. Pettifor, in Metallurgical Chemistry, edited by O. Kubashevski (HMSO, London, 1972).

${ }^{66}$ O. K. Andersen, J. Madsen, U. K. Poulsen, O. Jepsen, and J. Kollar, Physica B \& C 86-88B, 249 (1977).

${ }^{67}$ H. L. Skriver, Phys. Rev. B 31, 1909 (1985).

${ }^{68}$ F. Ducastelle and F. Cyrot-Lackmann, J. Chem. Phys. Solids 32, 285 (1971).

${ }^{69}$ P. Turchi and F. Ducastelle, in Recursion Method and Its Applications, edited by D. G. Pettifor and D. L. Weaire (Springer, New York, 1985).

${ }^{70}$ D. G. Pettifor, Bonding and Structure of Molecules and Solids (Clarendon Press, Oxford, 1995).

${ }^{71}$ A. V. Ruban, H. L. Skriver, and J. K. Nbrskov, Phys. Rev. Lett. 80, 1240 (1998).

${ }^{72}$ J. A. Moriarty, Phys. Rev. B 38, 3199 (1988).

${ }^{73}$ J. A. Moriarty, Phys. Rev. B 42, 1609 (1990). 